\title{
Identification of global modeshape from a few nodal eigenvectors using simple free-form deformation
}

Received: 11 August 2003/ Accepted: 4 February 2005/Published online: 14 July 2005

(C) Springer-Verlag London Limited 2005

\begin{abstract}
A new method, different from common eigenvalue extraction methods, was proposed by $\mathrm{Li}$ and Kikuchi (in 8th ARC conference, 2002). It consists of explicit finite-element method and eigenvalue-extraction method in time domain. Even though the new method performs well in extracting eigenvalues, it is difficult to identify global modeshape of the given structure due to large size of time history data. Only some eigenvectors of a few nodal points can be extracted. In this paper, we apply computer animation technique to identify the global modeshape from a few nodal eigenvectors. Freeform deformation (FFD) technique is simply modified - simple FFD - and applied to the identification of global modeshapes. The basic concepts that consist of simple FFD algorithm are Delaunay triangulation and barycentric coordinate. Some numerical examples show good performance for the identification of global modeshape of a given structure.
\end{abstract}

Keywords Eigenvalue $\cdot$ Modeshape $\cdot$ Computer animation - Delaunay triangulation - Barycentric coordinate $\cdot$ Free-form deformation

\section{Introduction}

There are various analytical eigenvalue extraction methods that are used in industrial and academic fields. Broadly, they are classified into two categories; one is the direct method and the other is the iteration method. Householder method and Jacobi method are good examples of the direct method [2]. The direct method is good to deal with small degrees of freedom. The iteration method is suitable for the models with large degrees

Y. Hahn $(\bowtie) \cdot$ N. Kikuchi

Department of Mechanical Engineering, University of Michigan, 2350 Hayward, Room 3005 EECS, Ann Arbor, MI 48109, USA

E-mail: yhahn@umich.edu

E-mail: Kikuchi@umich.edu of freedom. One of the common iteration methods is the Lanczos method [3]. Compared with other methods, the Lanczos method has certain advantages of fast convergence and simple multiplication. The Lanczos method is currently used as a standard eigenvalue solver of commercial engineering software.

However, the experimental method to extract eigenvalue is different from the analytical extraction method explained above. The analytical method is not employed in the experimental test because it is impossible to make a mass and stiffness matrix for the entire structure. In order to get eigenvalues experimentally, the object, attached with many sensors, is hit by the impact hammer. Using fast fourier transform (FFT), eigenvalues can be extracted from the time history data, which is obtained from the sensors. The time history data includes one of displacement, velocity and acceleration history data.

We can use the FFT or Ibrahim time domain method [4] to extract the eigenvalues from common small mechanical components in the experimental test because the output is just the transfer function in the impact test. However, for big objects, such as bridge and building, it is almost impossible to perform impact test due to noise generated from the environment. The special time domain extraction method-Stochastic Subspace Identification method [5] - is used to deal with such a big structure.

Similar to the experimental eigenvalue extraction procedure, a new numerical method to identify eigenvalues and eigenvectors of large-scale structure, which was based on the image-based finite element model, was proposed by $\mathrm{Li}$ and Kikuchi [1]. Using the special properties of image-based finite element model, the proposed method has an advantage in calculating the global mass and stiffness matrix. The same dimension of each element in the image-based model admits only a single calculation for one element to calculate the mass and global stiffness matrix.

The procedure of the new method proposed by $\mathrm{Li}$ and Kikuchi [1] is shown in Fig. 1. First, from the im- 
Fig. 1 Graphical procedure for the proposed eigenvalue extraction method

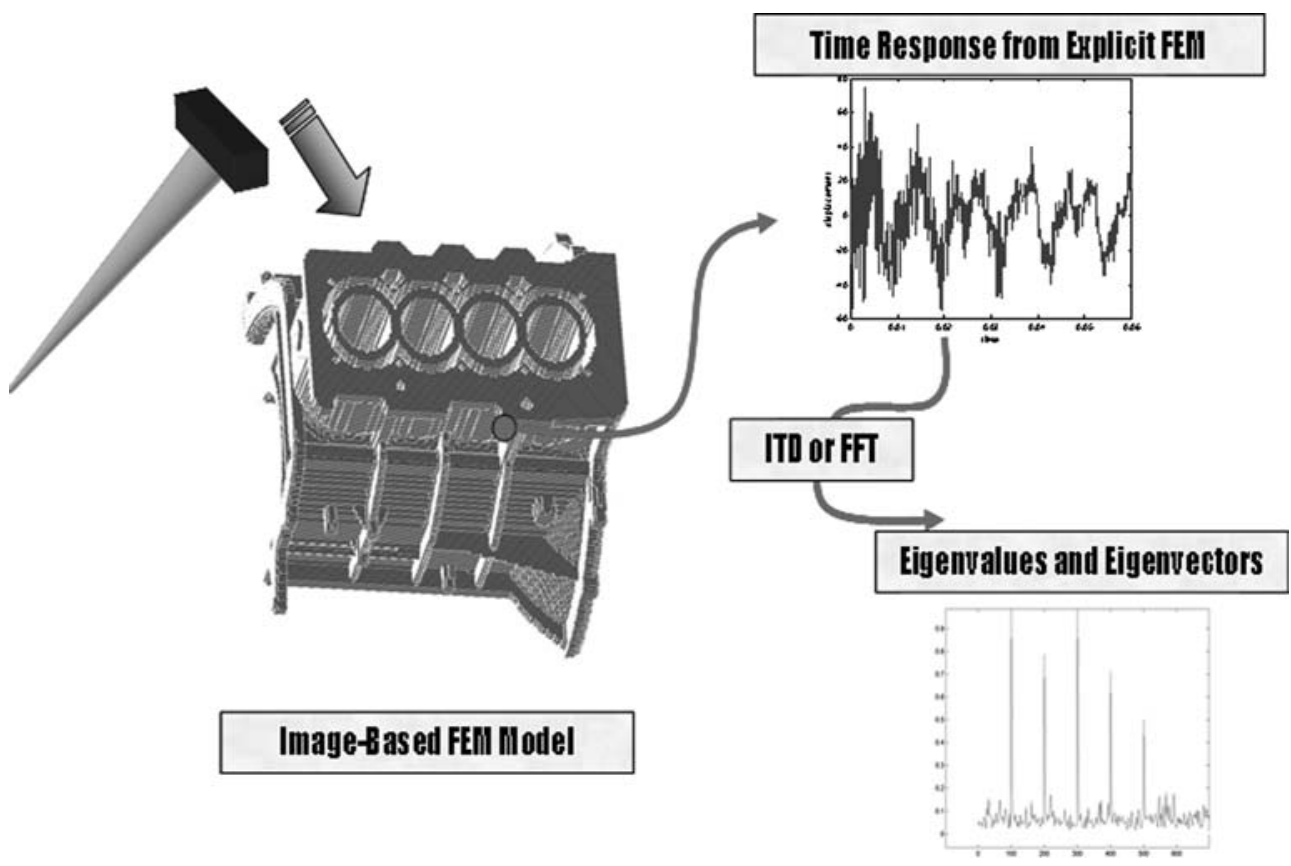

age-based finite element model, impact test is performed using explicit time integration method. Among various time integration methods, the modified Euler time integration method was chosen [6, 8]. For a consistent diagonal mass matrix, special element similar to Sauer's [7] was developed [8]. After obtaining the time response result, eigenvalues and eigenvectors can be obtained using Ibrahim time domain method [4] or FFT. However, due to the large-scale of the model and large storage size of history results, only some eigenvectors of a few nodal points can be obtained. It is hard to identify the global modeshape of the object using this proposed extraction method even though eigenvalues can be identified successfully.

In order to get the global modeshape of the object, we have to interpolate or extrapolate the given eigenvector

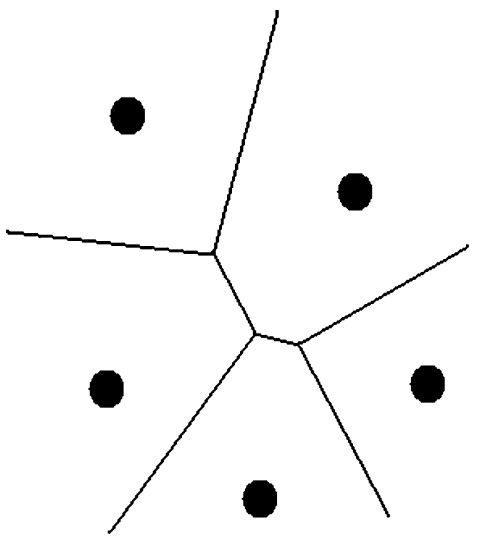

Voronoi Diagram

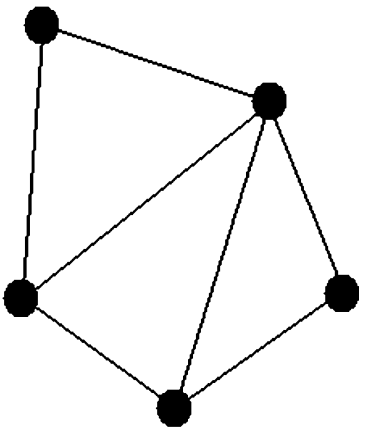

Delaunay Tessellation
Fig. 2 Voronoi diagram and Delaunay tessellation results from the proposed method. Interpolation or extrapolation technique can be found in computer animation field. In this research, free-form deformation (FFD) [9] and Dirichlet free-form deformation (DFFD) [10] are considered. From the basic concept of each computer animation technique, we developed a simple FFD - we call this method "simple FFD". Actually simple FFD is an already-known method and used for a long time in computer animation field; but, in this paper, we developed our own simple FFD that is suitable for our application.

Simple FFD consists of two processes; one is to make Delaunay triangulation for selected control nodes and the other is to set barycentric coordinate for all nodes except control nodes. Moreover, the concept of ghost control point is added for good performance. Using the simple FFD, global modeshape can be obtained successfully. Some examples show that the proposed method is proper for identifying the global modeshape. Even though the motion is not smooth, we can identify clearly which modeshape the object has.

\section{Basic mathematical terminology}

Before considering the computer animation techniques, some mathematical terminologies should be reviewed. These mathematical terminologies are widely used and useful in science and engineering fields.

\subsection{Voronoi diagram}

Figure 2 shows Voronoi diagram in 2-dimension. For $n$ given points in plane, Voronoi diagram can be defined as 
Fig. 3 Sibson coordinate

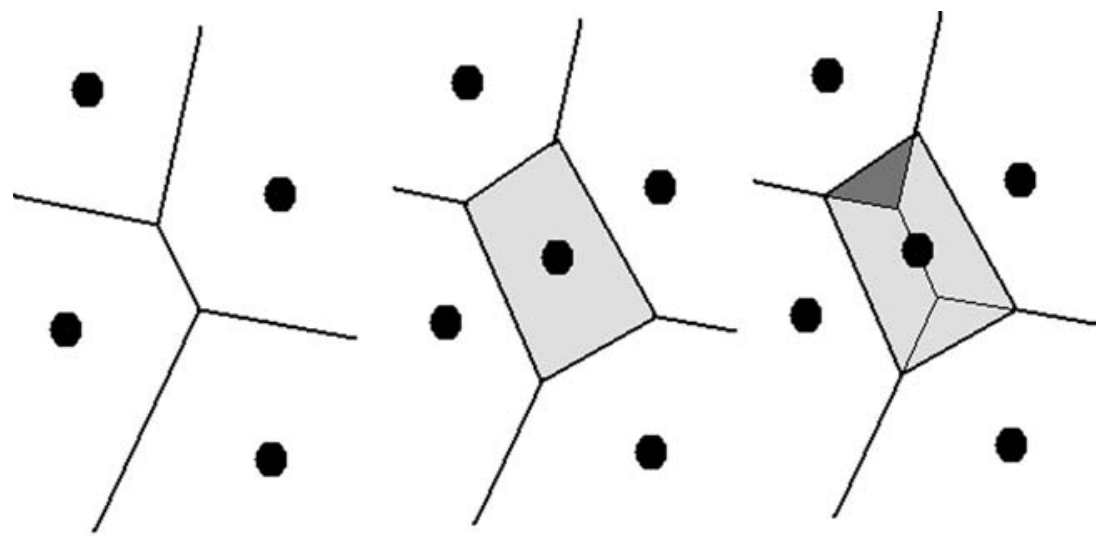

Contribution Factor : $\square / \square$

the polygons that have exactly one point. Each polygon is constructed by the lines are at the same distance from two adjacent points. Each polygon in Voronoi diagram is called a Voronoi cell. This 2-dimensional concept can be directly extended to $n$-dimensional case. This is one of the most important mathematical concepts in various fields because Voronoi diagram arises in many science and engineering fields [11].

\subsection{Delaunay tessellation}

Delaunay tessellation is the dual of Voronoi diagram. As shown in Fig. 2, Delaunay tessellation in 2-dimension is defined as triangles that consist of points inside of which the triangles are empty. It is also called Delaunay triangulation. This concept can also be extended to $n$-dimensional domain directly.

There are many methods to calculate Delaunay tessellation, for example, divide-and-conquer, sweepline algorithm, incremental algorithm, and convex hullbased algorithm [12]. For convenience, MATLAB is used in this research for calculation of Delaunay tessellation.

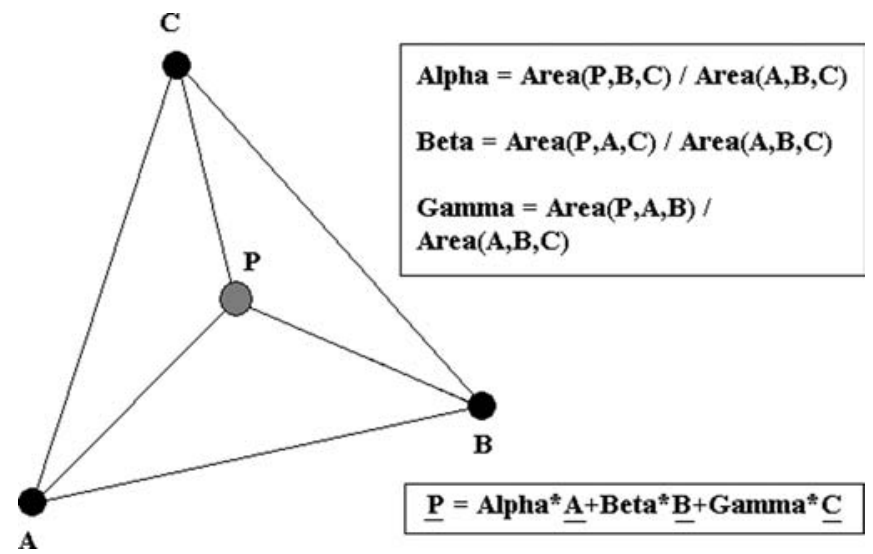

Fig. 4 Barycentric coordinate

\subsection{Sibson coordinate}

Sibson coordinate was introduced by Sibson in 1980 [13]. Sibson coordinate is shown in the Fig. 3; Voronoi diagram can be obtained from four points that already exist. If we insert another point, another Voronoi diagram for five points can be obtained. From two different Voronoi diagrams, the contribution factor of Voronoi diagram areas can be calculated. Using these contribution factors, Sibson coordinate system can be set. This concept can be extended to $n$-dimensional case.

Sibson coordinate is also called natural neighbor coordinate. Area-based coordinate, such as Sibson and barycentric coordinate, is more powerful than distancebased coordinate for multivariate data system [14].

\subsection{Barycentric coordinate}

This coordinate system is one of the oldest but still broadly used. This is a powerful coordinate system in the engineering field and easy to understand as shown in Fig. 4. Similar to the Sibson coordinate, the ratio of the lebesgue measure of two triangulations is used for the contribution factor. In the 2-dimensional case, the lebesgue measure of triangulation is the area of the triangle. In the 3-dimensional case, it is the volume of the tetrahedron.

\section{Computer animation technique}

Even though there are various computer animation methods [15], in this research, we focused on FFD which is one of the common computer animation techniques.

Free-form deformation was introduced by Sederberg and Parry in 1986 [9]. FFD starts to impose a local coordinate system on a parallelopiped region that consists of several control points. These control points can move to deform the object. Once the local coordinate is set to the object, each point in the object can be defined 

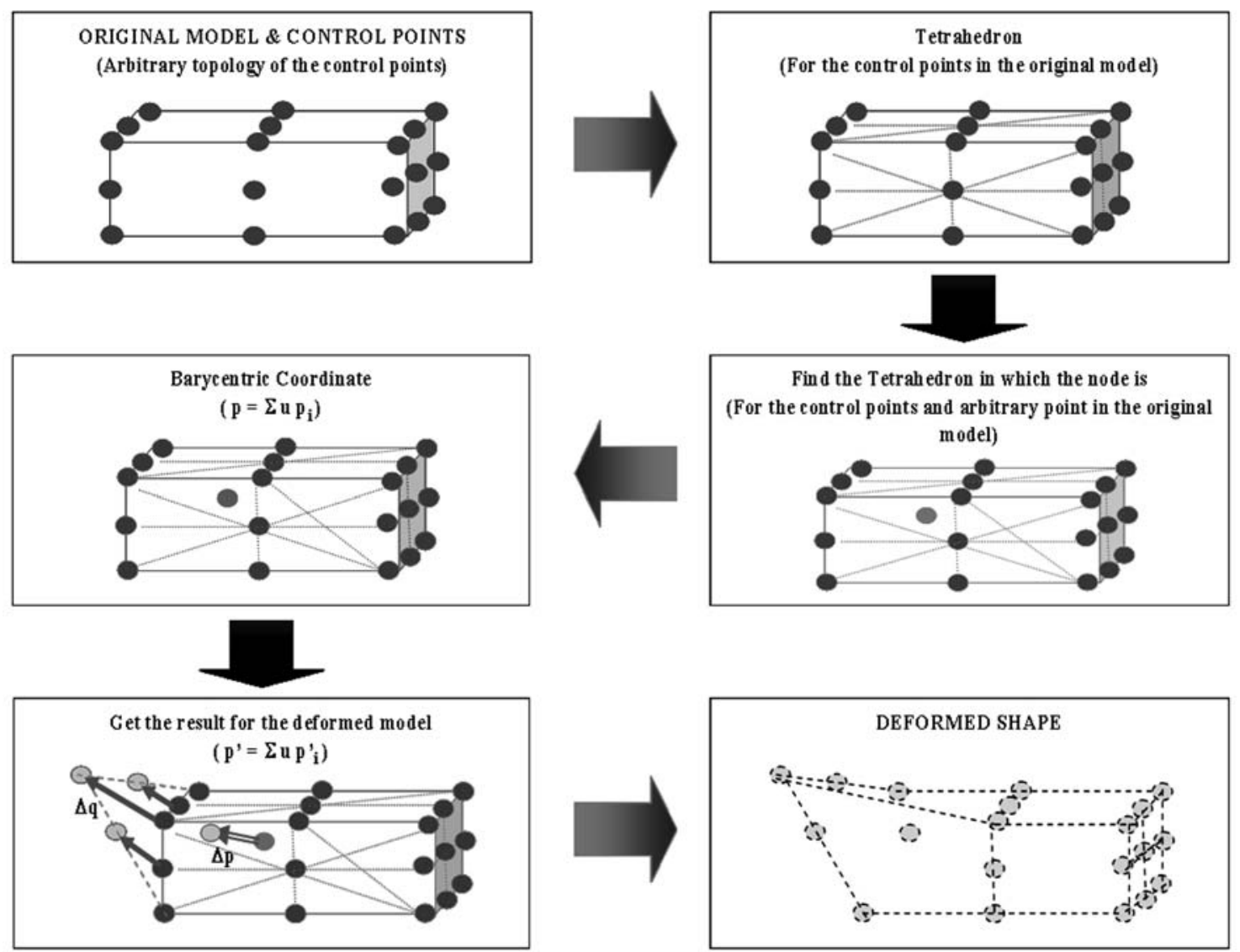

Fig. 5 Graphical procedure of the proposed method for identification of global modeshape from a few eigenvectors

using the control points as the coefficients of trivariate Bernstein polynomial. If new positions of control points are assigned, the coordinate values of the points in the object can be changed. However, FFD has the drawback that control points must be in a box shape to set local coordinate. This causes the limitation to control the detailed animation.
Many other animation techniques have tried to overcome the limitation of FFD. DFFD is one of the methods that are applied to overcome the limitation of FFD. DFFD was proposed by Moccozet and Thalmann [10] in 1997. Instead of trivariate Bernstein polynomial and rectangular coordinate, DFFD uses Sibson coordinate and Beizer surface. DFFD makes it possible to
Fig. 6 The calculation of the volume of the tetrahedron

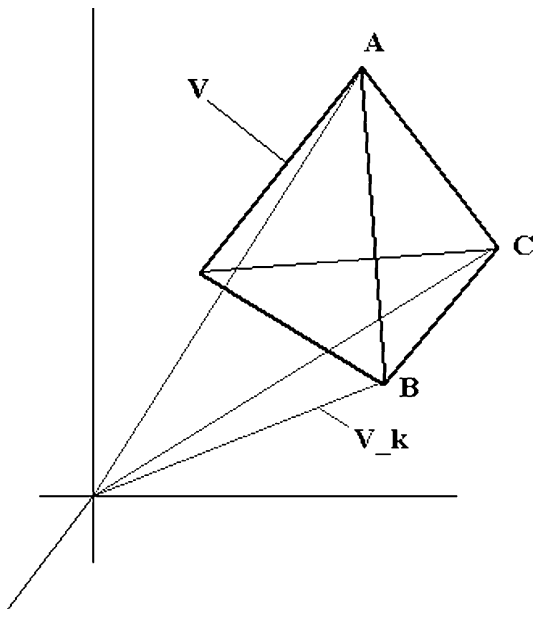




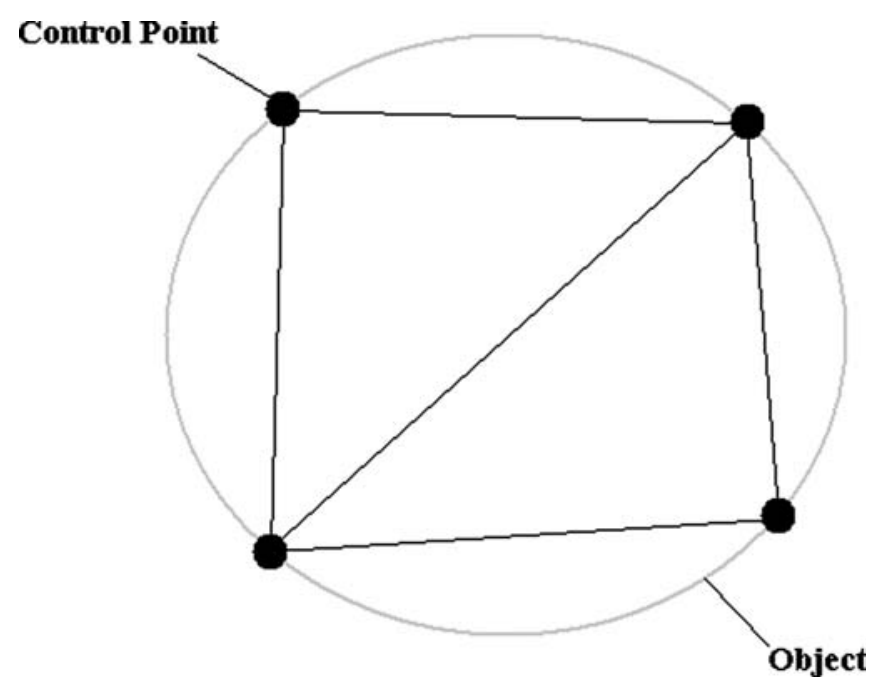

Fig. 7 The object that could not be detected by triangulation consisted of current control points

overcome the limitation of FFD. Moccozet applied this method to hand simulation in his paper [10].

The DFFD procedure starts to make a Voronoi diagram for the given control points. After inserting another data point, a new Voronoi diagram can also be made. Using the ratio of the lebesgue measure of two different Voronoi diagrams for given control points and data points, Sibson coordinate can be obtained. Once Sibson coordinate is set, we can calculate new positions of points in an object using the coordinate values of the deformed control points. Finally, the Beizer surface can be constructed using the coordinate values of the new data points [10]. However, the calculation of the lebesgue measure of Voronoi diagram is very complicated.

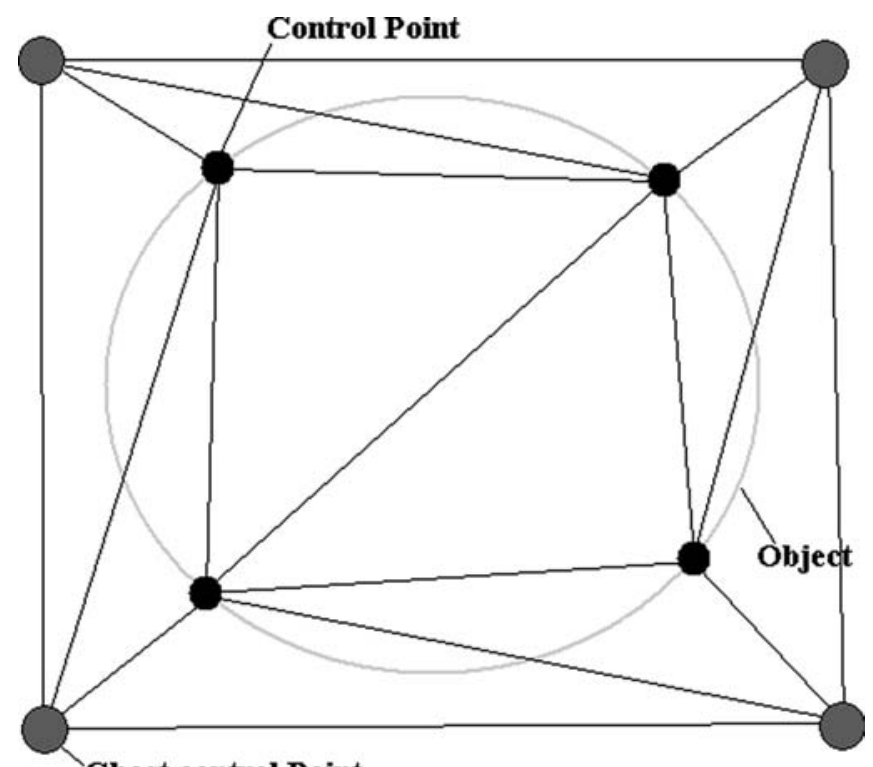

Ghost control Point

Fig. 8 The object that could be detected by triangulation consisted of current control points and ghost control points

\section{Proposed technique}

It is difficult to apply FFD or DFFD to identify the global modeshape from the given eigenvectors of a few nodes. There are two reasons. One is that the control points are not box shaped. There is no guarantee that control points are set in the parallelopiped shape, because the control points are arbitrarily selected. The other is that we have to deal with numerous nodes. Usually, the image-based finite element model has lots of nodes and elements. In this paper, we target the model of one million nodes and elements. Due to many nodes to deal with, a simple algorithm is needed.

In order to overcome the above problems, simple FFD is proposed. The proposed technique is the following as shown in Fig. 5:

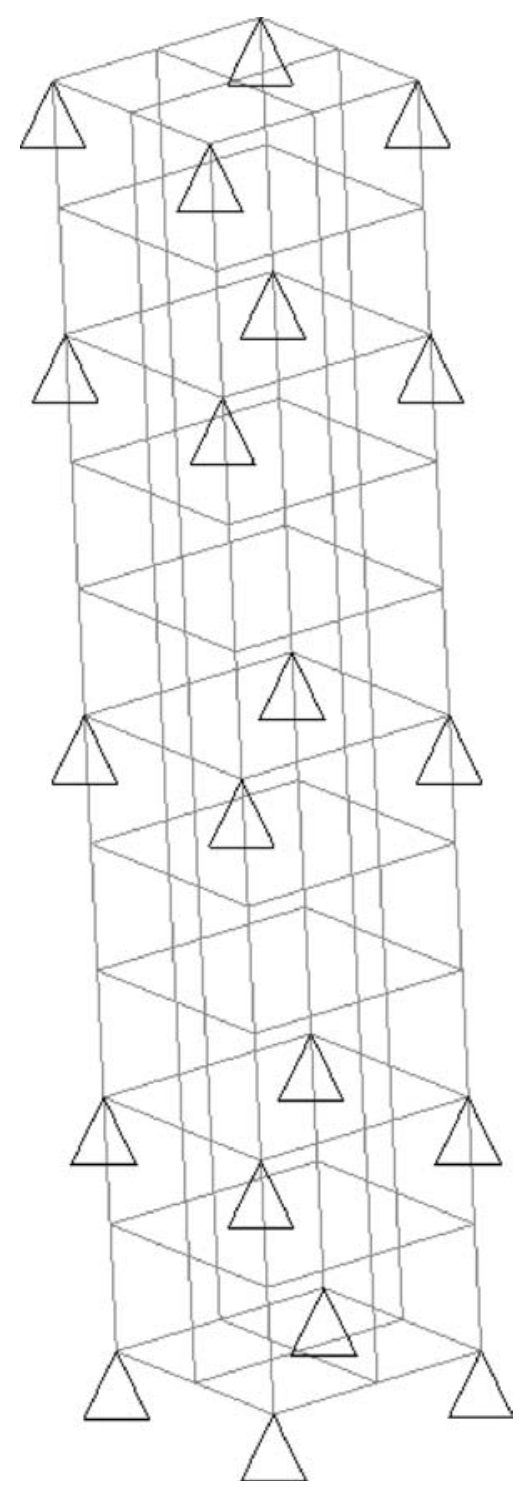

Fig. 9 Model and control points for simple beam model 
Fig. 10 The first modeshape a from a few eigenvectors using simple FFD and $\mathbf{b}$ from all eigenvectors
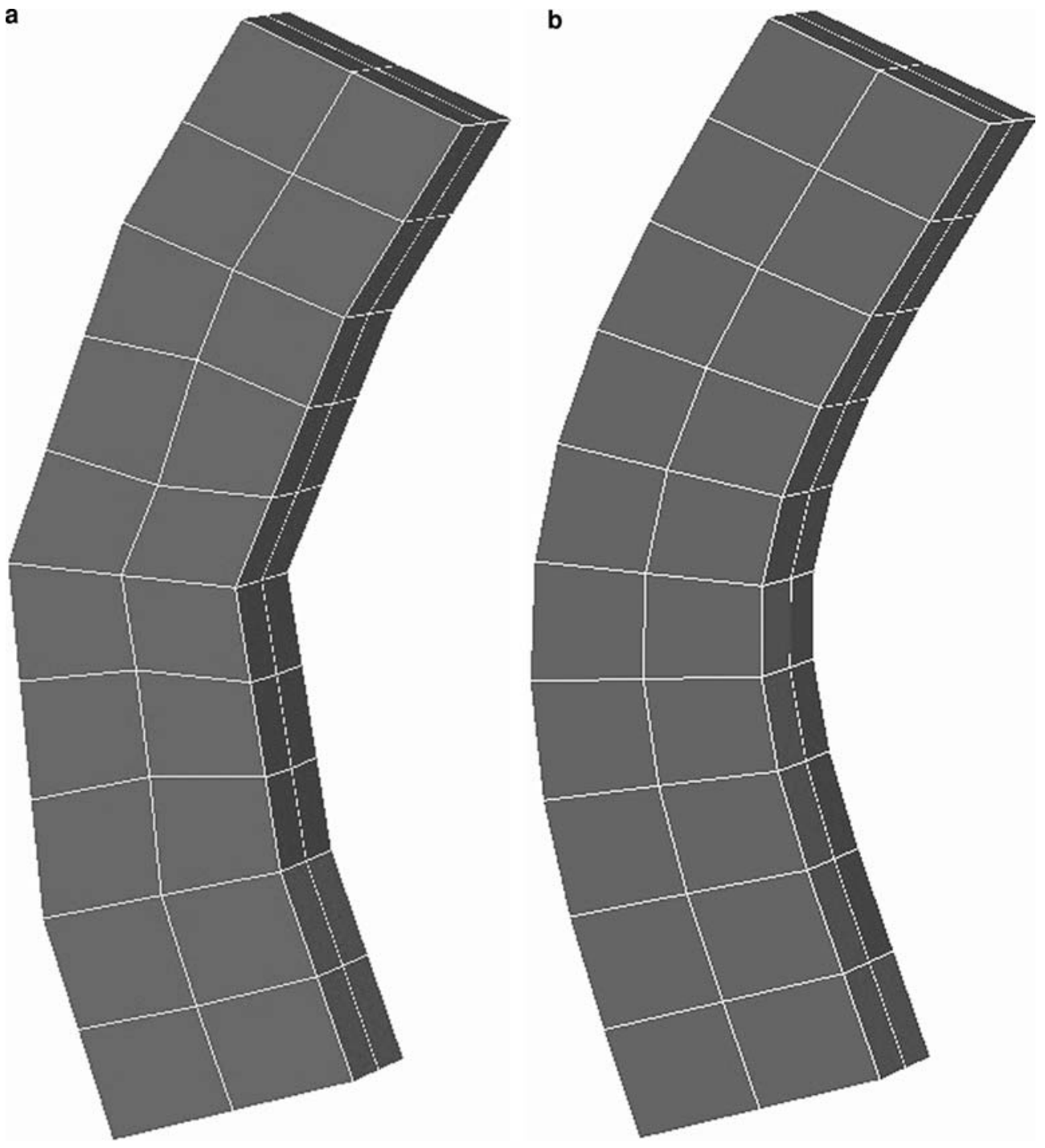

(a) A FE model and control points are constructed. The control points are output nodes from the new method [1]. Each control point has the eigenvector result from the finite element simulation. Other control points that are called "ghost control points" are also considered. Ghost control points are explained in the next section.

(b) For the given control points, tetrahedron can be constructed. For uniqueness, Delaunay triangulation is used.

(c) For each node that is not a control point in the FE model, it can be found out which tetrahedron contains that node.

(d) Barycentric coordinate can be set for each node.

(e) When the new coordinate value is assigned for control points, the new coordinate value can be obtained for each node.

(f) Repeating the process (e), we can get all eigenvectors of all nodes - global modeshape.
Figure 5 shows simple rectangular beam shape, but this procedure can be applied to any shape.

\section{Implementation issues}

There are two other issues for practical implementaion of this technique: one is to simplify the calculation process of the volume of the tetrahedron. The other is to set "ghost control points".

For the simplification of the volume calculation of the tetrahedron, the formula in Fig. 6 was used [16] in this research. It is simple to calculate and can be extended to $n$-polyhedron case. In Fig. $6, k$ indicates the index of the triangles that consists of polyhedrons.

Shown in Fig. 7, there are some parts in an object that cannot be detected through triangulation. To prevent this, the additional control points in Fig. 8 are considered. Extra four control points are defined outside the object. These additional control points do not exist 
Fig. 11 Model for a arbitrary shape solid model and $\mathbf{b}$ the control points on the model a
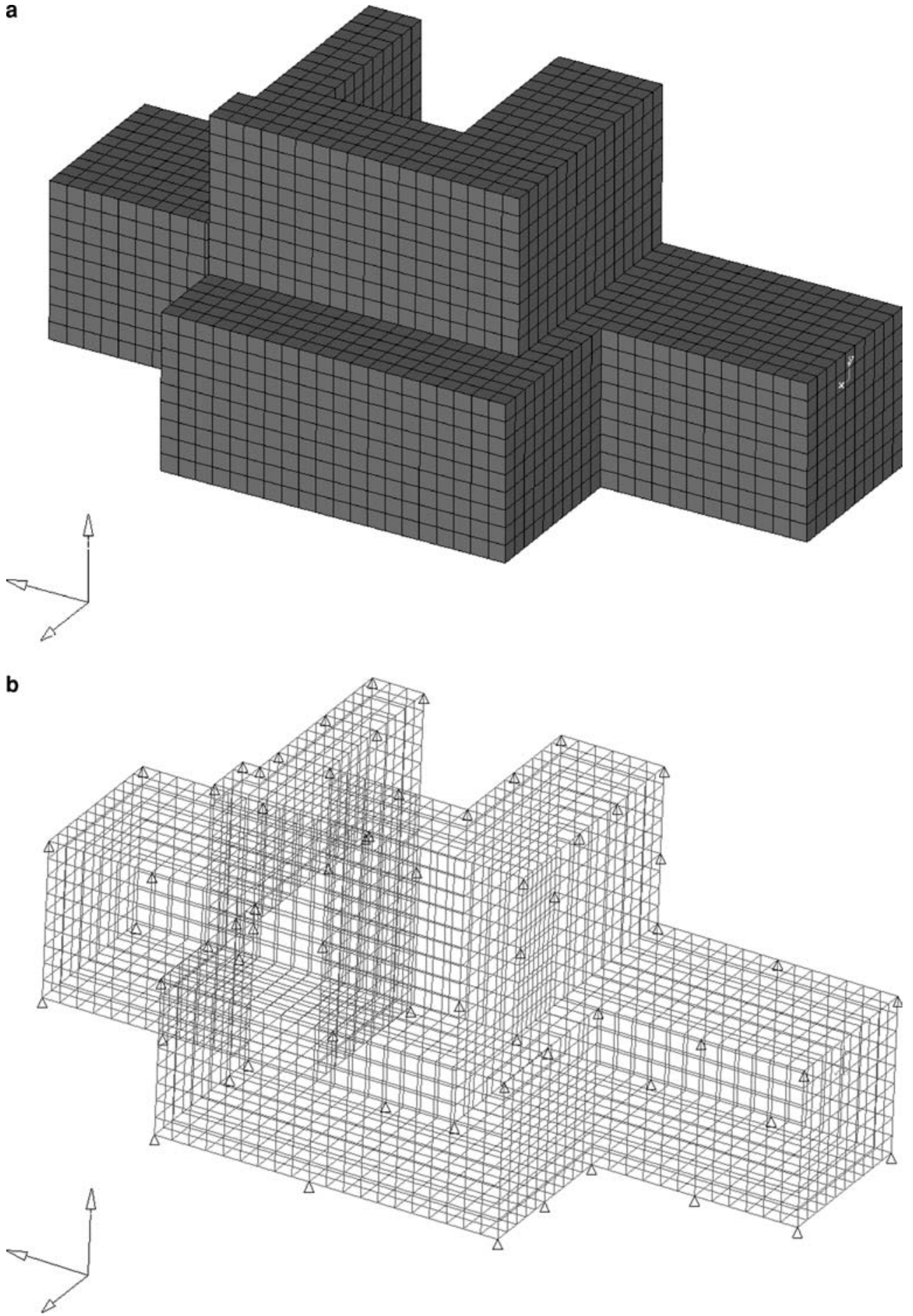

b in real. Using those extra points - which we call "ghost control points' in this paper-all points in the object can be detected as shown in Fig. 8.

\section{Numerical examples}

In order to verify the performance of the proposed method, the following seven examples are considered in this paper. The models of small degrees of freedom are used in the first four examples. In these examples, only first global modeshape was extracted, because other global modeshapes can be extracted in the same procedure. All simulation results were obtained by NASTRAN and post-processed by HyperMesh. Fifth example model has large degrees of freedom. In this paper, we chose the simple beam model that has about one million nodes and elements. The picture of the 
Fig. 12 The first modeshape a from a few eigenvectors using simple FFD and (b) from all eigenvectors
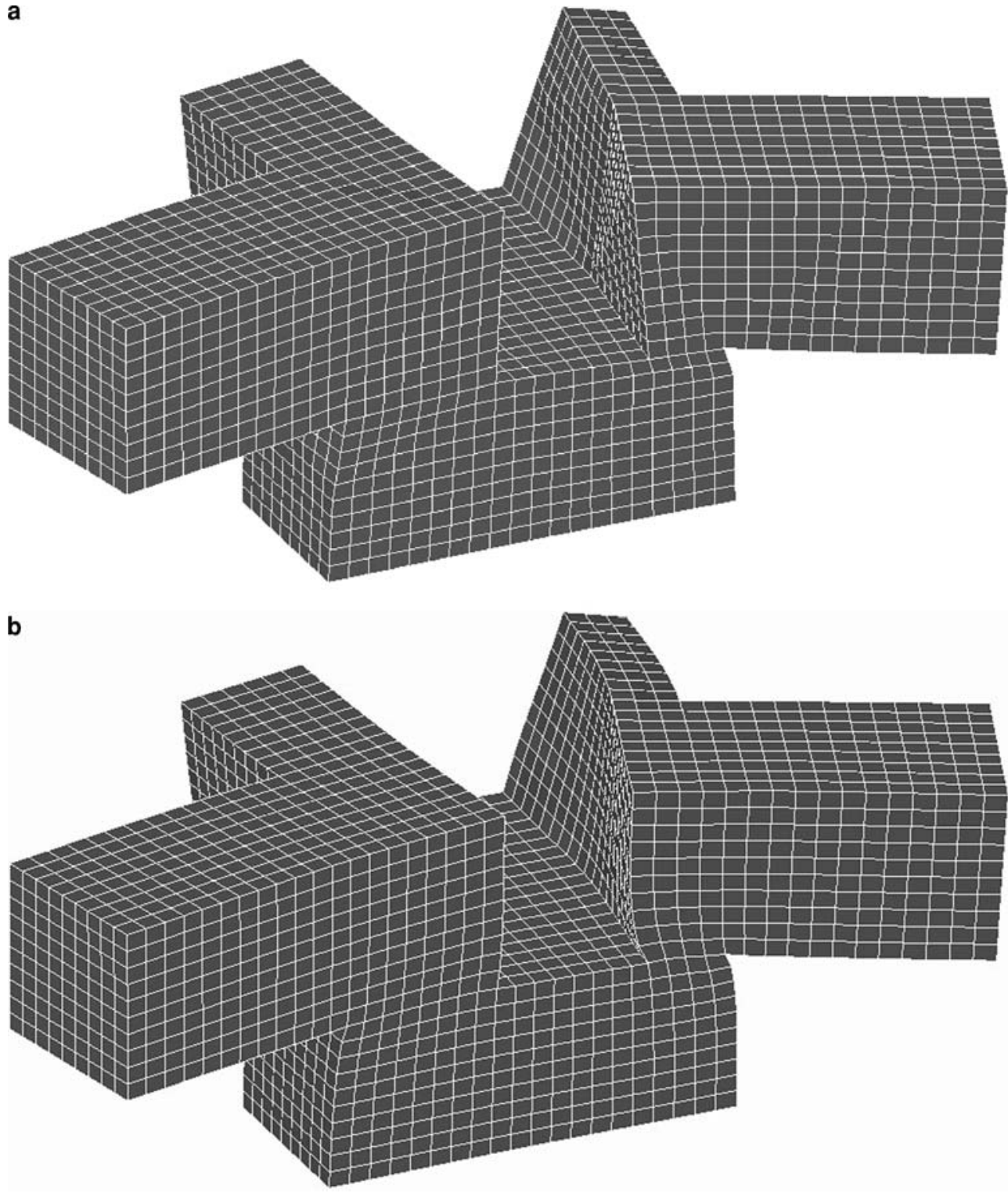

original model and control points for the fifth example are omitted because it is similar to the Example 1. This example is simulated by the new identification method that was proposed by Hong $\mathrm{Li}$ and Noboru Kikuchi [1] and post-processed by HyperMesh. The models of the last two examples are real mechanical components. One is automotive engine block and the other is Xerox shell panel. Both these models are image-based models. These examples are also performed by the new method [1] and post-processed by HyperMesh.

\subsection{Example 1: simple beam}

Figure 9 shows the simple beam model and control points. Each control point has its own eigenvector. This is the simple model for identification of global modeshape. Figure 10 shows the first global modeshape that comes from a few eigenvectors using simple FFD and from all eigenvectors. Comparing with the results in Fig. 10, we can identify that the first global modeshape is bending modes. Even though it is not smooth in Fig. 10a, we can recognize the same modeshape as shown in Fig. 10b.

\subsection{Example 2: arbitrary shape solid model}

Figure 11 shows the arbitrary shape of solid model and control points. Each control point has its own eigenvector. Figure 12 shows the first global modeshape from a few eigenvectors using simple FFD and from all eigenvectors. It is hard to identify the difference between 
Fig. 13 Model and control points for automotive bumper half model

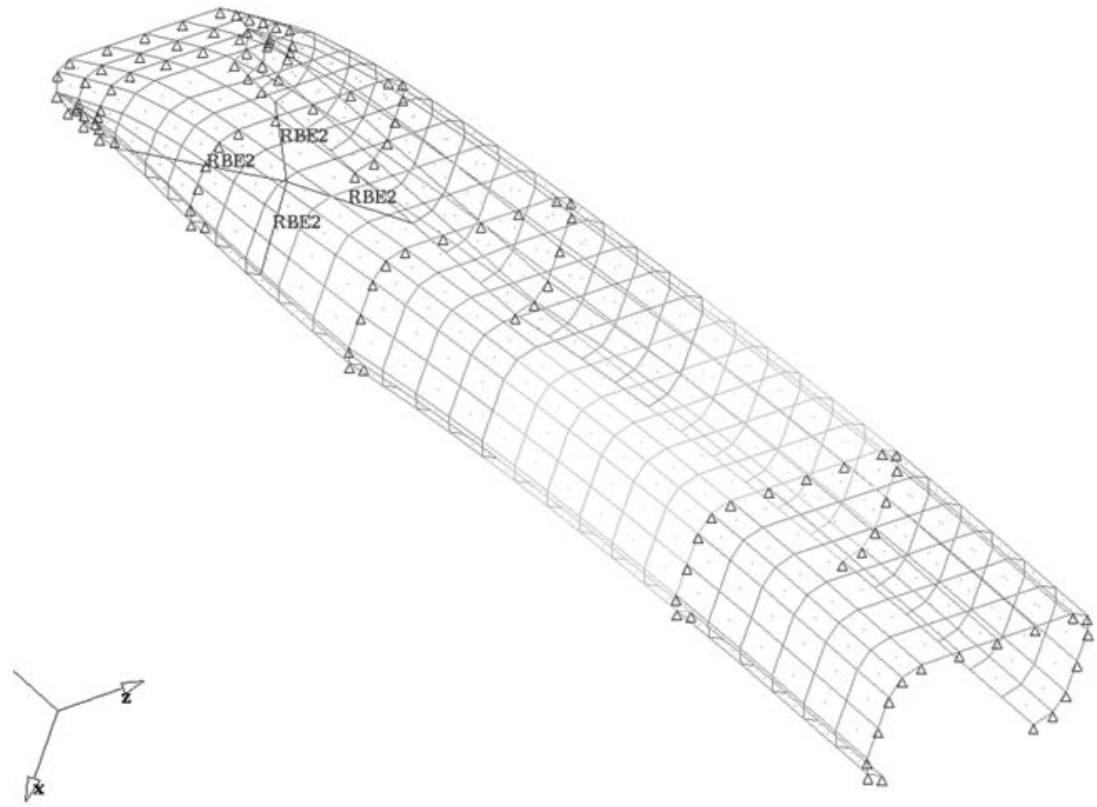

the two results in Fig. 12, due to the large number of control points.

\subsection{Example 3: half model of automotive} bumper-shell structure

Shell structure instead of solid structure is considered in this example. Figure 13 shows the half model of the automotive bumper and control points. Each control point has its own eigenvector. Four rigid elements are used to constrain the end motion in order to identify the modeshape clearly. Figure 14 show the first global modeshape from a few eigenvectors using simple FFD and from all eigenvectors. Similar to the previous examples, the modeshape can be identified successfully.

\subsection{Example 4: wire-type solid model}

This model of arbitrary curved solid wire-type structure is a good example for testing the proposed method in this paper. Figure 15 shows the model and control points. Each control point has its own eigenvector. In Fig. 15, there are many parts that cannot be detected by the tetrahedrons constructed by the control points. Using "ghost control point", they can be detected. Figure 16 shows the first global modeshape from a few eigenvectors and from all eigenvectors. In Fig. 16a, we can find out some strange deformations. But these deformations are caused by a small number of control points around that part. In the part that has many control points - middle part of the object - we can see the smooth deformation. Even though there are some strange deformations, we can identify the global modeshape successfully.

6.5 Example 5: large degree-of-freedom model-simple beam

The large model was tested and shown in Fig. 17. The model has about one million nodes and elements. It takes about $10 \mathrm{~min}$ to calculate the new positions of other nodal points from the 16 control points. Even though the shape is not smooth, the global modeshapes can be identified easily.

6.6 Examples 6 and 7: engine block model and xerox shell panel

The actual mechanical component models were tested. In Fig. 18, the results of the engine block and the Xerox panel model are shown. All results in Fig. 18 are first modeshape - torsional mode. Two models have about 200,000 to 300,000 nodes and elements.

\section{Conclusion}

A new eigenvalue extraction method was proposed by Hong Li and Kikuchi. The new method had a drawback that it was hard to identify the global modeshape due to the large number of mode size. In order to identify the global modeshape, two computer animation techniques are reviewed-FFD and DFFD. Considering computing cost, FFD technique is modified with barycentric coordinate and Delaunay triangulation-simple FFD. Several 
Fig. 14 The first modeshape a from a few eigenvectors using simple FFD and $\mathbf{b}$ from all eigenvectors

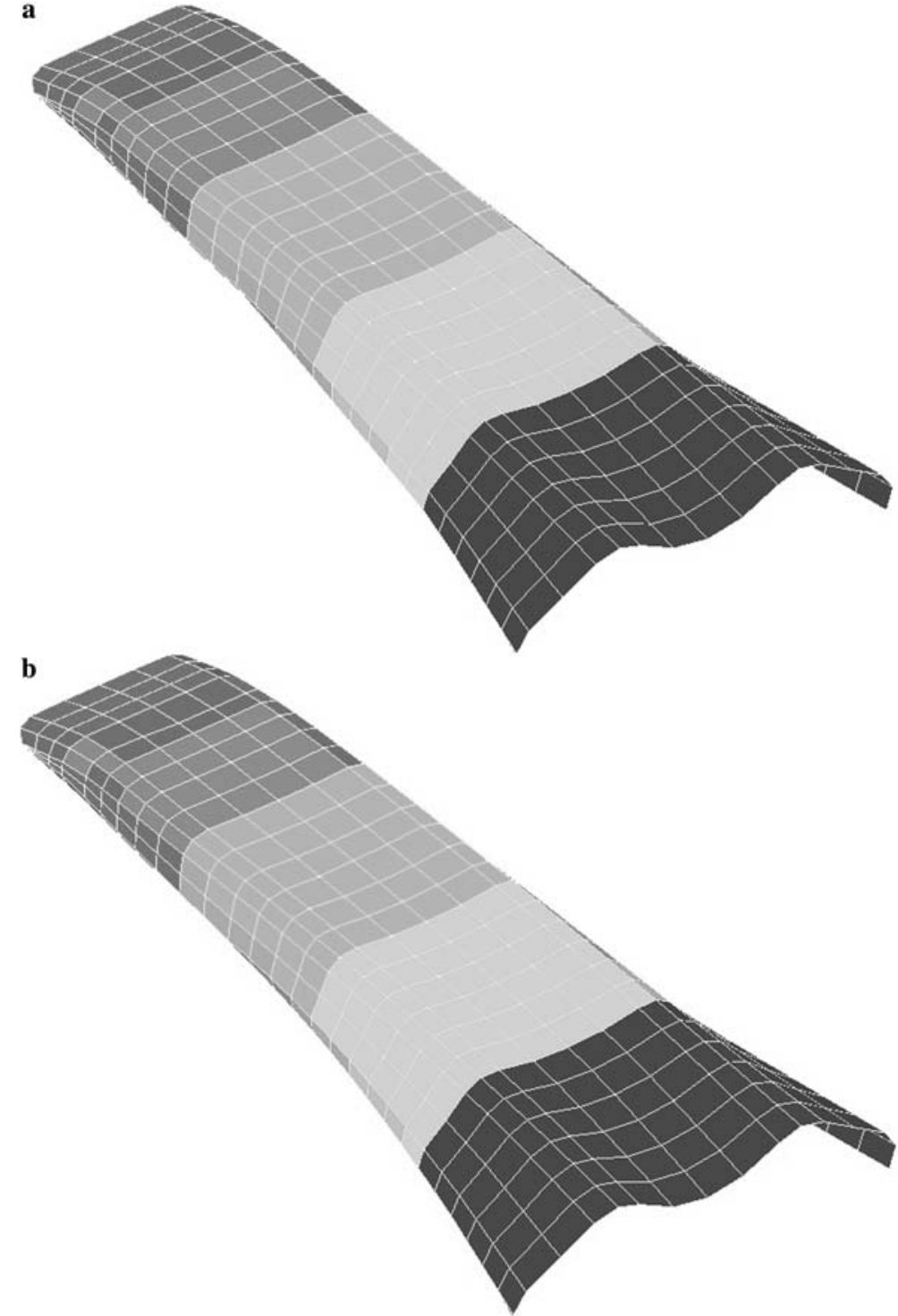

examples are tested for verification. For simple beam with one million nodal points, it takes approximately $10 \mathrm{~min}$ to get all eigenvectors of other nodes. We also applied simple FFD to real problems such as the engine block model and the Xerox panel model. Even though the deformation was not smooth, identification could be done successfully.

Acknowledgements The support of the Automotive Research Center is gratefully acknowledged. 


$$
h
$$


126

Fig. 16 The first modeshape a from a few eigenvectors using simple FFD and $\mathbf{b}$ from all eigenvectors
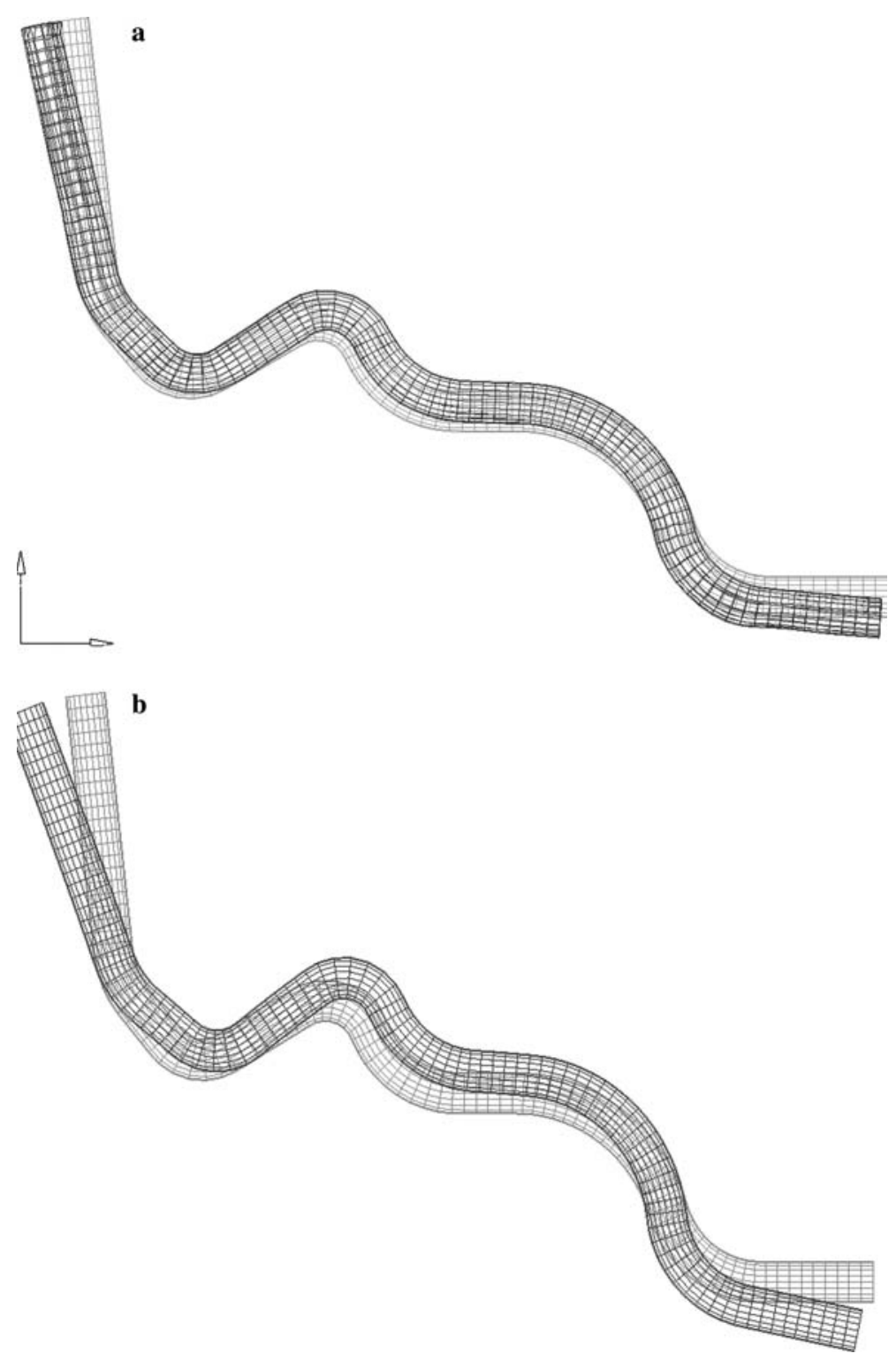
Fig. 17 Global modeshapes of the simple beam from a few eigenvectors [a first global modeshape (bending mode), b second global modeshape (torsion mode)] a

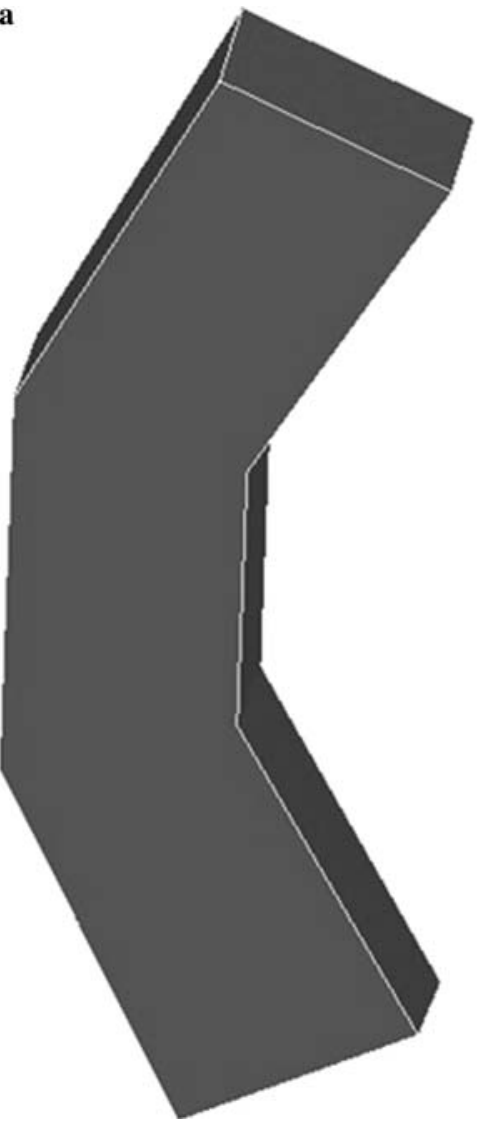

b

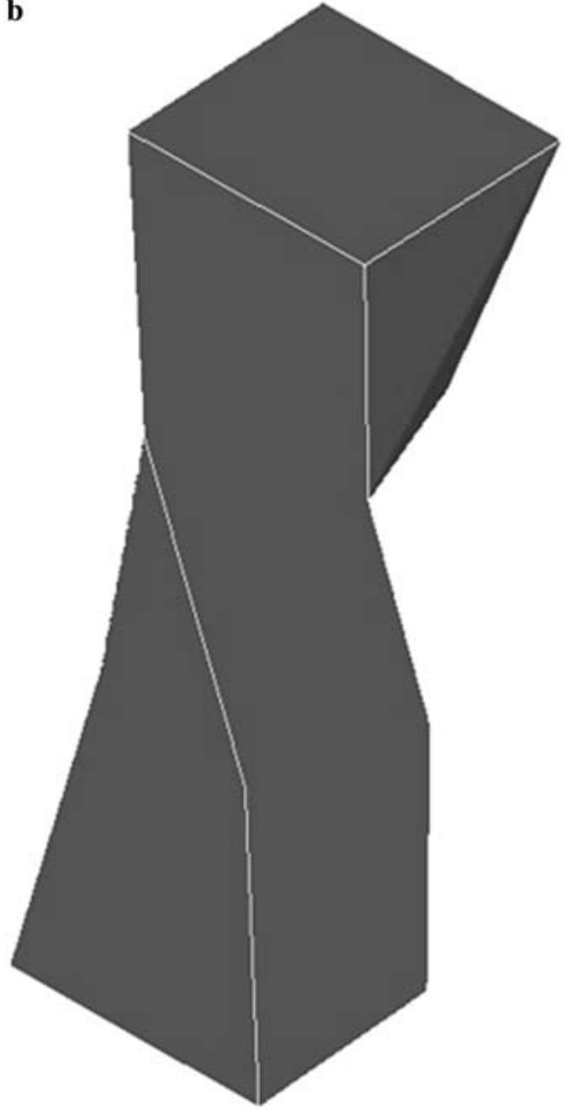


Fig. 18 First global modeshape of $\mathbf{a}$ engine block and $\mathbf{b}$ xerox shell panel, which are the result of a few eigenvectors
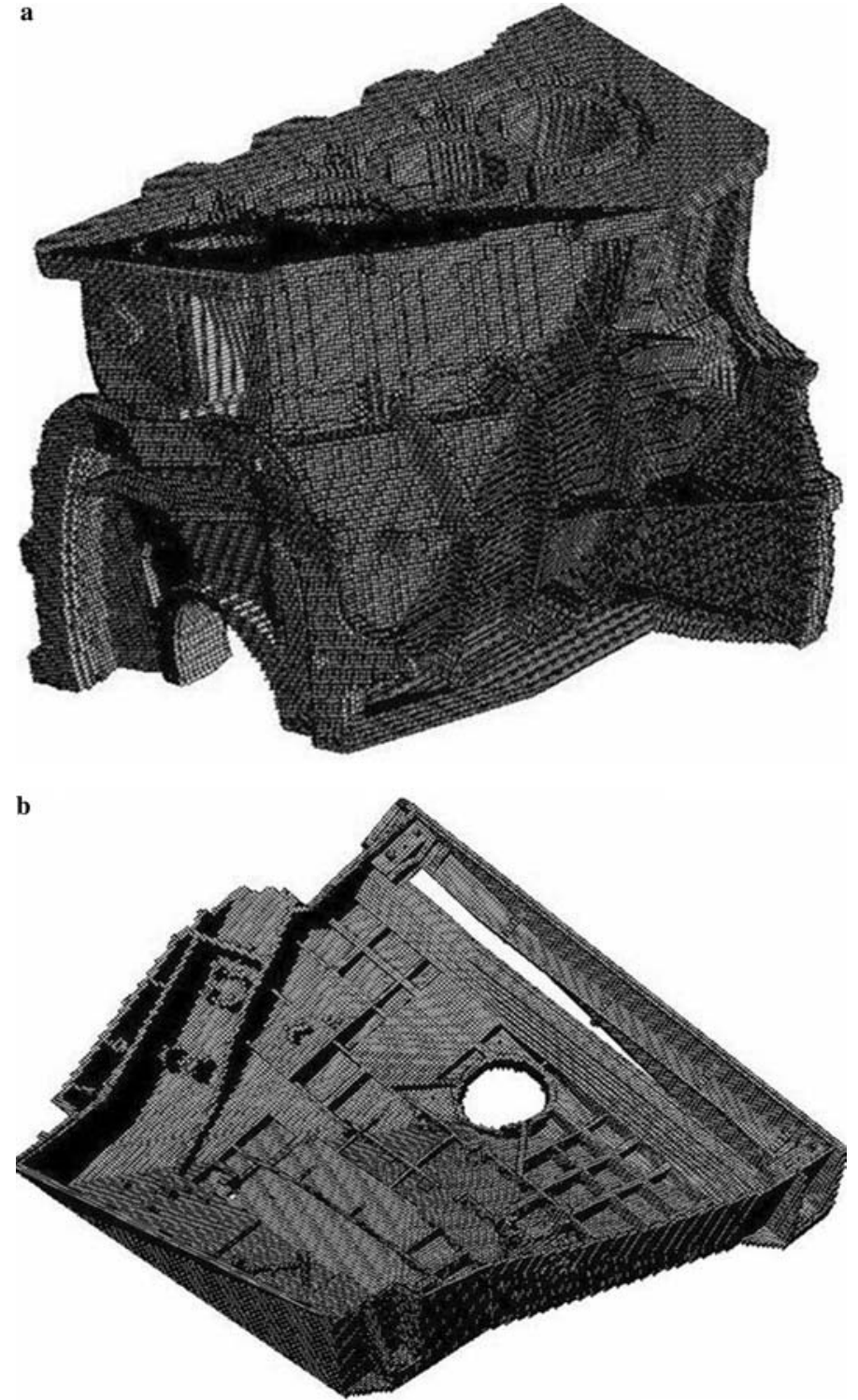

9. Sederberg TW, Parry SR (1986) Free-form deformation of solid geometric models. Comput Graph 20(4):151-160

10. Moccozet L, Thalmann NM (1997) Dirichlet free-form deformations and their application to hand simulation. In: Proceedings of Computer Animation 1997. IEEE Computer Society, pp 93-102.

11. Aurenhammer F (1991) Voronoi diagrams-a survey of a fundamental geometric data structure. ACM Comput Surv 23(3):345-405

12. Welzl E, Su P, Scot Drysdale RL III (1997) A comparison of sequential Delaunay triangulation algorithms. Comput Geom $7: 361-385$

13. Sibson R (1980) A vector identity for the Dirichlet tessellation. Math Proc Camb Phil Soc 87:151-155

14. Watson DF (1992) Contouring: a guide to the analysis and display of spatial data. Pergamon Press, New York

15. Vince J (1992) 3-D computer animation. Addison-Wesley, Reading

16. Zhang C, Chen T (2001) Efficient feature extraction for $2 \mathrm{D} / 3 \mathrm{D}$ objects in mesh representation. ICIP 2001. Thessaloniki, Greece
8. Li H (2002) A new approach to the identification of eigenmodes of large structures. PhD Dissertation, University of Michigan 